\title{
DEATH-STAR: Silicon and Photovoltaic Fission Fragment Detector Arrays for Light-Ion Induced Fission Correlation Studies
}

\author{
J.D. Koglin ${ }^{\mathrm{a}, \mathrm{b}, *}$, J.T. Burke ${ }^{\mathrm{b}}$, S.E. Fisher ${ }^{\mathrm{b}}$, I. Jovanovic ${ }^{\mathrm{c}}$ \\ ${ }^{a}$ Pennsylvania State University, University Park, PA 16802, United States \\ ${ }^{b}$ Lawrence Livermore National Laboratory, Livermore, CA 94550, United States \\ ${ }^{c}$ University of Michigan, Ann Arbor, MI 48109, United States
}

\begin{abstract}
The Direct Excitation Angular Tracking pHotovoltaic - Silicon Telescope ARray (DEATH-STAR) combines a series of 12 silicon detectors in a $\Delta E-E$ configuration for charged particle identification with a large-area array of 56 photovoltaic (solar) cells for detection of fission fragments. The combination of many scattering angles and fission fragment detectors allows for an angular-resolved tool to study reaction cross sections using the surrogate method, anisotropic fission distributions, and angular momentum transfers through stripping, transfer, inelastic scattering, and other direct nuclear reactions. The unique photovoltaic detectors efficiently detect fission fragments while being insensitive to light ions and have a timing resolution of $15.63 \pm 0.37 \mathrm{~ns}$. Alpha particles are detected with a resolution of $35.5 \mathrm{keV} 1 \sigma$ at $7.9 \mathrm{MeV}$. Measured fission fragment angular distributions are also presented.
\end{abstract}

Keywords: fission-fragment detector, photovoltaic cell, solar cell, surrogate reaction, angular distribution

\section{Introduction}

Many nuclear reaction experiments require accurate detection of heavy ions in the presence of a large background of light ions. Scattered beam

5 can be large source of this background for measurements of the angular distributions of fission fragments at forward angles; additionally, nuclides with large alpha decay rates can hinder the detection of their fission fragments. In the study of light-ion re-

10 actions, silicon telescope detectors are ubiquitously used to identify $p, d, t,{ }^{3} \mathrm{He}$, and $\alpha$ exit channels, among others. The use of photovoltaic (solar) cells enables detection of heavy charged ions while being insensitive to the light ion backgrounds that are 15 unavoidable in many scattering experiments. The Direct Excitation Angular Tracking pHotovoltaicSilicon Telescope ARray (DEATH-STAR) combines an array of silicon telescope detectors with an array of photovoltaic cells for fission fragment detection. 20 Counting and identification of charged-particle exit channels as a function of scattering angle is achieved

\footnotetext{
${ }^{*}$ Corresponding author

Email address: koglin1@llnl.gov (J.D. Koglin)
}

Preprint submitted to Nuclear Instruments and Methods A via an array of silicon $\Delta E-E$ telescopes, while angular-dependent counting of fission fragments is performed using a large array of small photovoltaic 25 cells.

\section{Photovoltaic Array}

The use of photovoltaic cells for fission fragment detection was first demonstrated by Siegert [1 in 1979. Subsequently, the energy spectrum of ${ }^{252} \mathrm{Cf}$ 30 was accurately measured using solar cells 2, 23. Since then, solar cells have been used to tag fission events for prompt gamma-ray spectroscopy [4], as fission fragment-sensitive targets for spectroscopy of prompt fission-fragment X-rays [5, and to com35 pensate for large coincident gamma-ray signals [6]. A detection system combining silicon telescopes with fission detectors has been used to measure the ${ }^{238} \mathrm{Pu}(n, f)$ cross section [7].

\subsection{Photovoltaic Detectors}

40 Commercial solar cells are not intended for use as fission fragment detectors and care is required when selecting cells for this purpose. Many are manufactured with a thin anti-reflective coating on the

February 15, 2017 


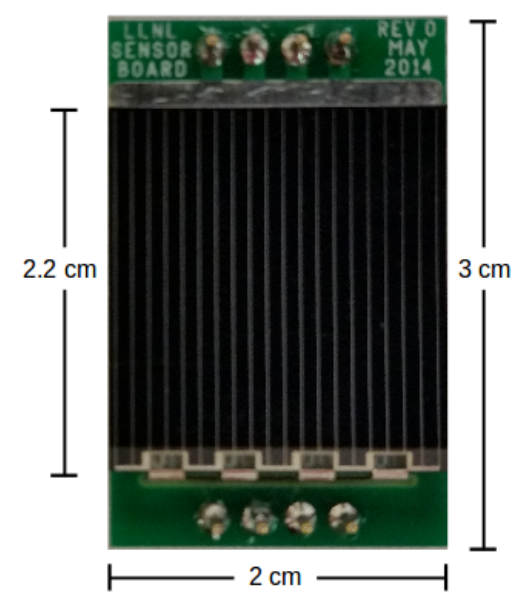

Figure 1: Solar cell mounted to the PCB. Pins extending from the back of the board plug into the support structure and allow for easily swapping detectors.

front surface of the silicon to increase photon ab45 sorption, but the coating acts as a dead layer for heavy ions. It is more common for older models of solar cells to lack the anti-reflective coating, as was the case with the cells employed in this work. Simulations performed by Hsieh [8] suggest that subso strates with high resistivity and high capacitance are desirable to maximize charge collection. High resistivity, however, increases the parallel noise and causes the pulse rise time to increase.

Photovoltaic cells used for DEATH-STAR con55 sist of a $2 \times 2.2 \mathrm{~cm}^{2}$ area of $80 \mu \mathrm{m}$-thick monocrystalline silicon layer on a $20 \mu \mathrm{m}$-thick silver backing produced by the company Solar Made. They are mounted on custom printed circuit boards (PCBs) to provide rigidity, as shown in Figure 1 1 . The ${ }_{60}$ front of the PCB contains a large conductive pad, to which the silver back of solar cell is attached using a vacuum compatible acrylic adhesive embedded with silver particles to provide conduction. The front contact of the photovoltaic is threaded 65 through a slot in the PCB and is similarly attached to a conductive pad on the back of the PCB. The front and back conductive pads are connected to positive and negative connection pins, respectively. This board is plugged into a second PCB "mother70 board", which is mounted on a steel support structure and wired for readout. In this way, the photovoltaic cells can be easily replaced when damaged and rearranged. A hollow steel sphere provides the support structure for the photovoltaic array. The 75 silicon telescopes are positioned at the equator and define the reaction plane. Photovoltaic cells are located at 56 positions $15^{\circ}$ above and below the equator in $10^{\circ}$ polar intervals. Moving toward the pole in $10^{\circ}$ polar intervals, 20 detectors are spaced at so $15^{\circ}$ azimuthal intervals, then 20 detectors at $18^{\circ}$ azimuthal intervals, and finally 12 detectors are placed at $30^{\circ}$ azimuthal intervals.

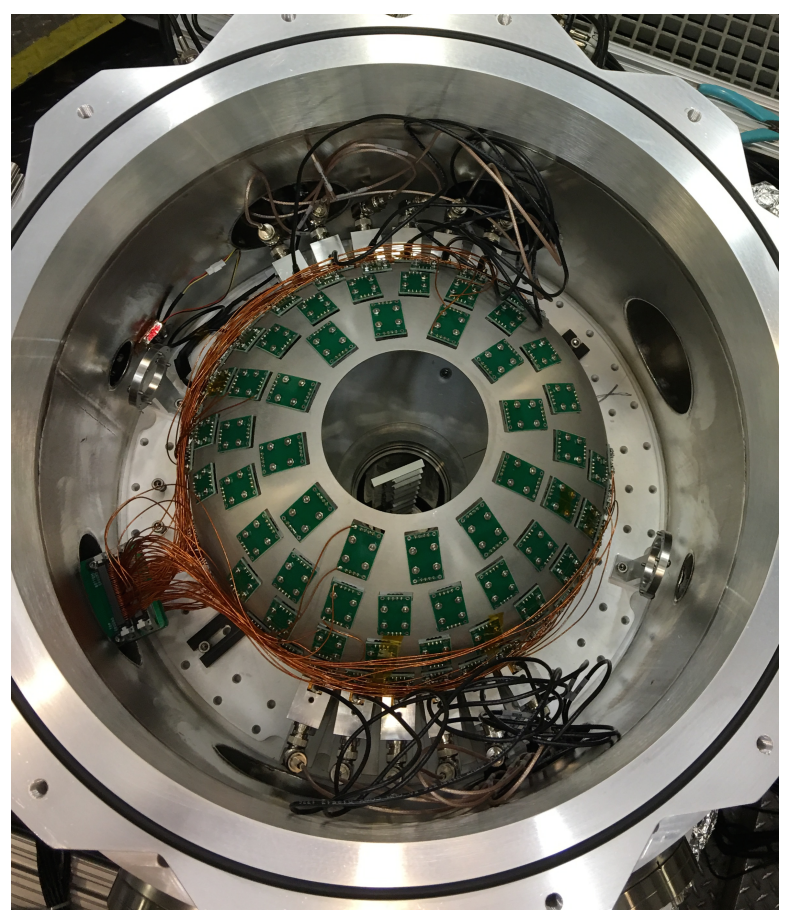

Figure 2: The steel scattering chamber contains the spherical mounting structure for the photovoltaic array. A row of photovoltiac detectors exists below the silicon telescopes that cannot be seen in this figure. The target arm is rotated $45^{\circ}$ with respect to the beam and extends through the bottom of the chamber.

\subsection{Field Funneling Effect}

The ability for photovoltaic cells to function as effective fission fragment detectors in the presence of large field of light ions relies on the field-funneling effect. Simply, when a charged particle ionizes a track trough a depletion region created by an n$\mathrm{p}$ junction the electric field is distorted into the so underlying neutral substrate and enhances charge collection. The field funneling effect is strongly dependent on the ionization density such that fission fragment tracks produce a large charge pulse and those from $\alpha$ particles are indistinguishable from 


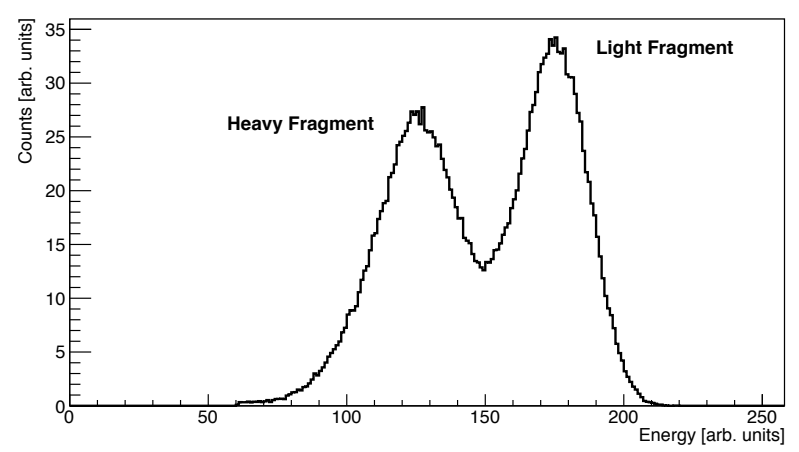

Figure 3: Fission fragment energy spectrum measured from spontaneous fission of ${ }^{252} \mathrm{Cf}$. The heavy and light fragment peaks are clearly separated.

noise. This effect is discussed in detail in References [8, 9, 10.

\subsection{Energy Response}

The energy response of the photovoltaic cells ${ }_{150}$ was measured using a thin, electroplated $1436 \mathrm{~Bq}$ ${ }^{252} \mathrm{Cf}$ source positioned $\sim 7 \mathrm{~cm}$ from the center of the cell, producing a $0.8 \mathrm{cps}$ rate in $2 \mu$ Torr vacuum. The cells were operated without bias; the positive terminal was connected to the input of a Swan Research $100 \mathrm{mV} / \mathrm{MeV}$ CHARGE8V chargeintegrating preamplifier 11. The resulting spectrum is shown in Figure 3 . The separation of the light and heavy fragment peaks is clearly evident and the entire spectrum is well separated from lowenergy noise. Schmitt et al. [12] have previously developed criteria to quantify the performance of silicon semiconductor fission fragment detectors. These criteria are not specific for detectors utilizing the funneling mechanism, but they can be used to compare the performance of photovoltaic cells to traditional silicon semiconductor detectors. The Schmitt parameters have been calculated based on the parameters defined in Figure 4 and are listed in Table1 for the spectrum measured in Figure 3 . The expected values listed in Table 1 are derived from "averages from some of the better performing detectors" 12. Spectrum parameters 1-3 are indicative of the overall detector resolution, with parameter 1 being the most sensitive due to the relatively sharp peak produced by the lighter fragments. The photovoltaic cell meets the expected value for parameter 2 , but performs worse for parameter 1 . This is attributed to the dependence of the funneling effect on ionization density; the heavier fragments have a denser ionization track, which enhances the charge
30 collection and improves the resolution for the respective peak. Parameters 4 and 5 are higher order checks of overall detector resolution, and are the ones for which the solar cells have similar performance to traditional semiconductor detectors. Parameters $6-8$ provide a sensitive indication of lowenergy tailing of the fragment peaks, for which the solar cells do not meet any of the reasonable limits. A cause of this is incomplete charge collection from trapping in the substrate and reformation of the depletion layer, which stops the funneling process before all of the track charge has been collected. This is exacerbated by the lack of source collimation; different thicknesses of dead layers are encountered by fission fragments at different incident angles. It is clear that solar cells have relatively poor energy resolution compared to traditional silicon semiconductor detectors which are better suited for precise fission fragment spectroscopy. The photovoltaic detectors are better suited for high efficiency fission fragment counting in the presence of a large background of light ions where the advantages of the field-funneling effect outweigh the decrease in energy resolution.

\subsection{Pulse Shape and Timing}

Typical rise times were observed to be within 100 ns of $\sim 1 \mu \mathrm{s}$ by examining pulse shape at the output of $100 \mathrm{mV} / \mathrm{MeV}$ CHARGE8V chargeintegrating preamplifiers. A representative pulse is shown in Figure 5. The fast component arises from the funneling effect, while the slow component arises from diffusion of charge through the substrate to the anode. The relatively long rise time and undershoot are attributed to the $\gtrsim 40 \mathrm{nF}$ capacitance of the solar cells, which exceeds the rating

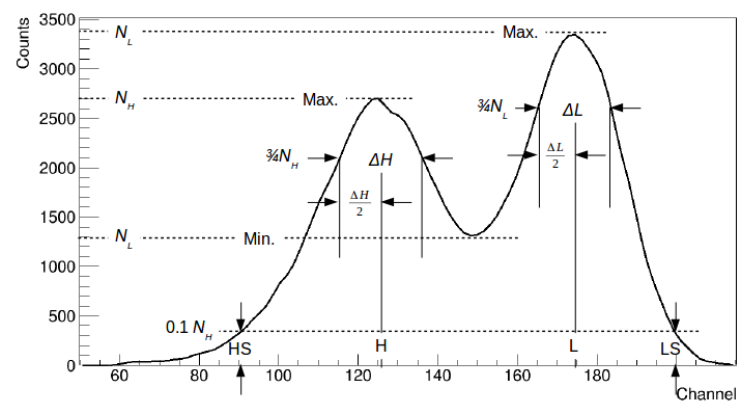

Figure 4: Smoothed ${ }^{252} \mathrm{Cf}$ fission fragment spectrum. The spectrum parameters for detector performance evaluation shown here have been introduced by Schmitt et al. 12 . 
Table 1: Parameters of the ${ }^{252}$ Cf fission fragment spectrum measured using a solar cell detector.

\begin{tabular}{|c|l|c|c|c|}
\hline No. & $\begin{array}{c}\text { Spectrum } \\
\text { Parameter }\end{array}$ & $\begin{array}{c}\text { Reasonable } \\
\text { Limit* }\end{array}$ & $\begin{array}{c}\text { Expected } \\
\text { Value* }^{*}\end{array}$ & $\begin{array}{c}\text { Measured } \\
\text { Value }\end{array}$ \\
\hline 1 & $N_{L} / N_{V}$ & $>2.85$ & $\sim 2.9$ & 2.72 \\
\hline 2 & $N_{H} / N_{V}$ & $\sim 2.2$ & $\sim 2.2$ & 2.2 \\
\hline 3 & $N_{L} / N_{H}$ & - & $\sim 1.30$ & 1.23 \\
\hline 4 & $\Delta L /(L-H)$ & $<0.38$ & $\sim 0.36$ & 0.38 \\
\hline 5 & $\Delta H /(L-H)$ & $\lesssim 0.45$ & $\lesssim 0.44$ & 0.44 \\
\hline 6 & $(H-H S) /(L-H)$ & $<0.70$ & $\lesssim 0.69$ & 0.75 \\
\hline 7 & $(L S-L) /(L-H)$ & $\lesssim 0.49$ & $\lesssim 0.48$ & 0.52 \\
\hline 8 & $(L S-H S) /(L-H)$ & $\lesssim 2.18$ & $\sim 2.17$ & 2.27 \\
\hline
\end{tabular}

*From Schmitt et al., Ref. [12]

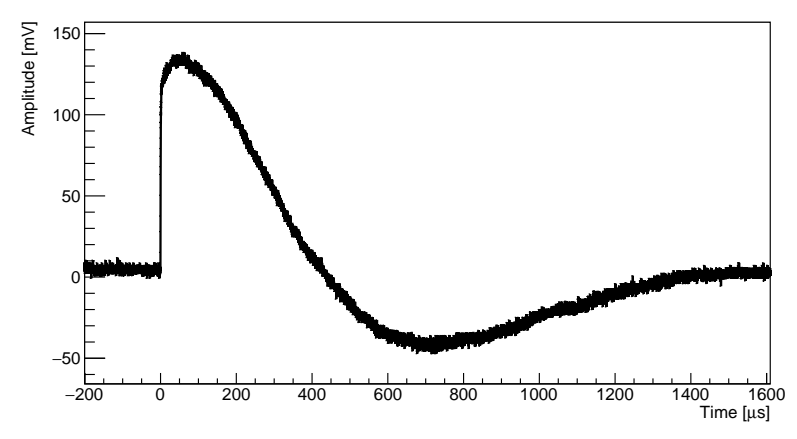

Figure 5: Typical pulse produced by interaction of a fission fragment with the photovoltaic detector after preamplification.

of the preamplifier. Although design improvements for charge-integrating preamplifiers have been reported [13, commercial chips are usually rated for detector capacitances $<1 \mathrm{nF}$. Exceptionally fast rise times have been reported for similar photovoltaic fission fragment detectors using specially designed, current-mode preamplifers. The SAPhIR detector 190 reported rise times of approximately $20 \mathrm{~ns}$ using specifically designed preamplifiers 44; experiments at the Los Alamos Neutron Science Center reported $50 \mathrm{~ns}$ rise time for their photovoltaic detectors using the same preamplifiers [6]. For fast-timing ap- 195 plications the charge-integrating preamplifiers used here are not sufficient. A measurement of the timing resolution was performed using an electrodeposited ${ }^{252} \mathrm{Cf}$ source sandwiched between thin mylar sheets, through which the fission fragments es- 200 cape. Cells were positioned a $1 \mathrm{~cm}$ from both sides of the source. The timing spectrum was recorded with an Ortec 927 multichannel analyzer. A timing resolution of $15.63 \pm 0.37$ ns FWHM was achieved, as indicated in Figure 6, despite the relatively slow 205

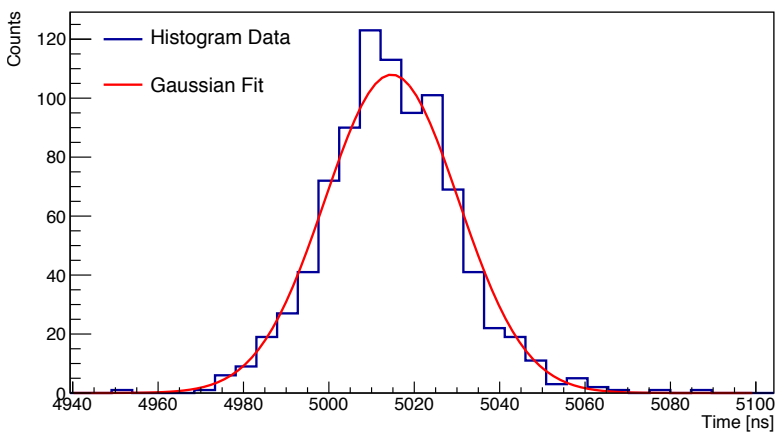

Figure 6: TAC spectrum of coincident fission fragments from ${ }^{252} \mathrm{Cf}$ spontaneous fission recorded by the photovoltaic cells.

rise time.

\subsection{Noise}

For best performance, the photovoltaic detectors are operated without bias. Applying a reverse bias does not improve detector resolution or increase pulse height. A large leakage current develops with small increases in bias. For these cells, each volt of applied bias results in approximately $1 \mu \mathrm{A}$ increase in the leakage current, which increases the associated noise and negates any benefits that applying a bias may give [1. The large capacitance of the detectors also makes them highly susceptible to electromagnetic noise pickup. Extreme care was needed to eliminate ground loops and adequately shield the signal wires and preamplifier housing. Additionally, it was necessary to remove vacuum pumps and switching power supplies that broadcast large electromagnetic signals from the experimental area. 


\section{Silicon Telescope Array}

\subsection{Scattering Chamber}

The cylindrical scattering chamber that holds the detector system is has a height of 12" and a 17.5" inner diameter. The base plate is patterned with two concentric rings of 36 screw holes spaced at $10^{\circ}$ intervals, with the beam exit defining $0^{\circ}$. These secure optical mounts to point directly toward the center of the chamber. Silicon detectors are attached to the optical mount with a custom holder positioning them at $15.72 \mathrm{~cm}$ from the chamber center. A schematic of the detector positions inside the scattering chamber is given in Figure 7 Signal cables are connected to BNC vacuum feedthroughs clustered in groups of 12 at four ports on the chamber. A fifth port was custom made to accommodate four 34-pin ribbon cable feedthroughs. Two 8 " printed circuit boards each, with two straightthrough 34-pin headers at either end, pass through two small slots in the flange and are sealed with Torr-Seal low vapor pressure epoxy to maintain vacuum. A micro CCD camera, $1 \mathrm{~cm} \times 1 \mathrm{~cm}$, has been installed inside the chamber with $5 \mathrm{~V}$ power and video signals carried on $\mathrm{BNC}$ connections to monitor luminous phosphors mounted on the target arm used for beam tuning. To prevent overheating while at vacuum, the camera is fixed to the scattering chamber wall using thermally conductive tape to act as a heat sink.

\subsection{Silicon Telescopes}

The particle telescopes consist of two silicon detectors. A thin, $\Delta E$ detector allows transmission of high energy light ions is backed by a thick, $E$ detector in which the ions are completely stopped. ferential energy loss. The $E$ detectors are circular Ortec L series lithium drifted silicon detectors 14. The active face of the detector has a diameter of $16.0 \mathrm{~mm}$ with an active depth of $5 \mathrm{~mm}$, and is capable of fully stopping $25 \mathrm{MeV}$ protons or $100 \mathrm{MeV}$ $\alpha$ particles. The $\Delta E$ detectors are circular Ortec B series totally depleted silicon surface barrier detectors [15]. The active thickness varies in the range of 170-215 $\mu \mathrm{m}$ between detectors. The active face diameter is $13.8 \mathrm{~mm}$, corresponding to $5.28^{\circ}$ angular spread and $0.048 \%$ solid angle per telescope $15.72 \mathrm{~cm}$ from the chamber center. A $16 \mu \mathrm{m}$ aluminum shield is placed in front of the $\Delta E$ detector to protect the silicon from damaging $\delta$-rays and fission fragments. Typically, telescopes are positioned 280

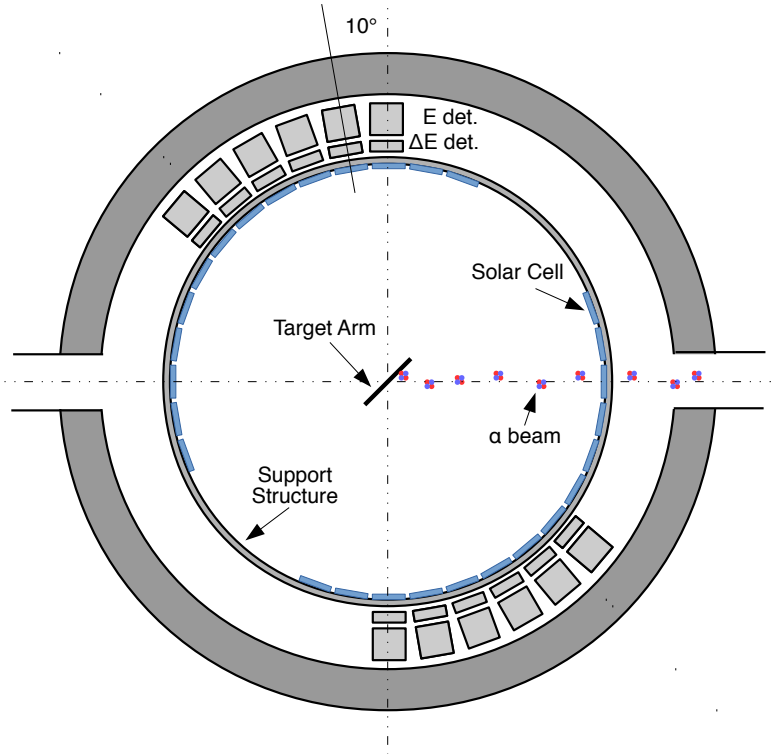

Figure 7: Schematic of the scattering chamber. Rings of solar cells are positioned above and below the plane of the $\Delta E-E$ telescopes. Gaps in the support structure give the silicon detectors a line of sight to the target.

downstream between $10^{\circ}$ and $90^{\circ}$ and on the opposite side of the chamber between $-90^{\circ}$ and $-170^{\circ}$.

\subsection{Targets and Target Arm}

A translatable target arm extends up through the bottom of the chamber at the center of the silicon array. It accommodates up to 12 targets and can change positions under vacuum. The target arm is rotated such that the targets lie at $45^{\circ}$ with respect to the beam axis to minimize straggling at the positions of interest. The target frames are the same as those used in the former STARS/LiBerACE array [16], having dimensions of $2.54 \mathrm{~cm} \times 3.81 \mathrm{~cm}$ with notches at the bottom to secure them to the target arm. Many positions on the arm are often hold luminous phosphor targets to facilitate with beam tuning. Other positions hold targets for calibration and contaminant corrections such as carbon, ${ }^{208} \mathrm{~Pb}$, and mylar. Typical actinide targets consist of a few hundred $\mu \mathrm{g} / \mathrm{cm}^{2}$ plutonium or uranium electroplated on a thin $\left(100 \mathrm{\mu g} / \mathrm{cm}^{2}\right)$ carbon backing.

\subsection{Resolution and Particle Identification}

The silicon detectors are each calibrated offline using the $4784.34(25), 5489.48(30), 6002.35(9)$, and $7686.82(7) \mathrm{keV}$ [17 $\alpha$ lines from a spectroscopy 
grade ${ }^{226}$ Ra source. Typical $1 \sigma$ energy resolutions for $\Delta E$ detectors are $\approx 35.5 \mathrm{keV}$ at $7.7 \mathrm{MeV}$. An energy spectrum from a $\Delta E$ detector obtained from a ${ }^{226} \mathrm{Ra}$ source is given in Figure 8. When considering particles other than $\alpha$ particles, corrections must be made for the pulse height deficit in silicon. Elastic and inelastic scattering reactions in-beam on carbon and ${ }^{208} \mathrm{~Pb}$ are used to calibrate the $E$ detectors above $7.7 \mathrm{MeV}$. Energy loss through the target and detector dead layers is estimated using the Energy Loss and Straggling Tool (ELAST) or The Stopping and Range of Ions in Matter (SRIM). Particles are identified by comparing the differential

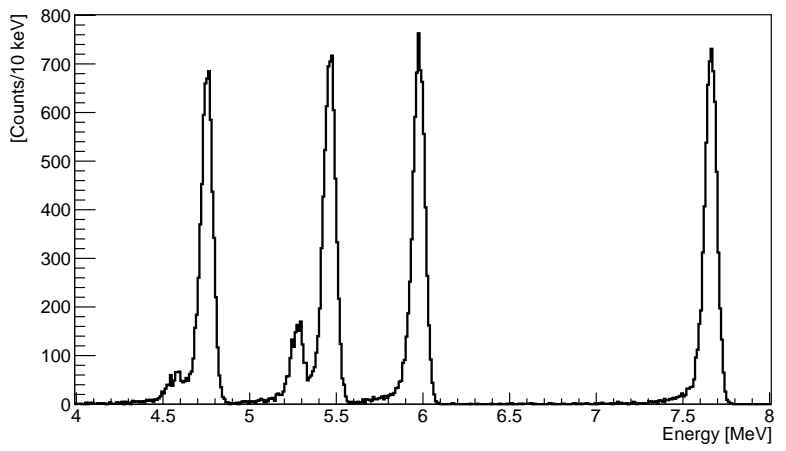

Figure 8: Measured ${ }^{226} \mathrm{Ra} \alpha$ spectrum for a typical $\Delta E$ detector. The $1 \sigma$ energy resolution at $7.7 \mathrm{MeV}$ is $35.5 \mathrm{keV}$.

energy loss in the $\Delta E$ detector to the energy of the particle [18] deposited in the $E$ detector. An example particle identification plot is shown in Figure 9 for the $36.9 \mathrm{MeV}$ alpha particles incident on ${ }^{240} \mathrm{Pu}$. There is exceptional separation between the alpha 335 band and the lighter ions. Improved separation between the proton, deuteron, and triton bands can be achieved by adjusting the ADC parameters. A significant amount of ${ }^{3} \mathrm{He}$ production is not seen in this reaction. An alpha spectrum measured in coin- 340 cidence with fission fragment detection is presented in Figure 10, alpha particles are detected at $90^{\circ}$ and $-90^{\circ}$. Fission is measured as the sum over many angles, specifically those shown in Figure 11. The fission decay channel opens at approximately $4.7 \mathrm{MeV}$ and the number of fissions observed increases up 345 to the neutron separation energy at which point the neutron emission decay channel competes with fission and gamma emission; the observed number of fissions decreases. The background spectrum is determined from non-prompt coincidences and 350 subtracted from the prompt coincidence spectrum. The horizontal error bars represent the bin widths which are adjusted in accordance with limitations

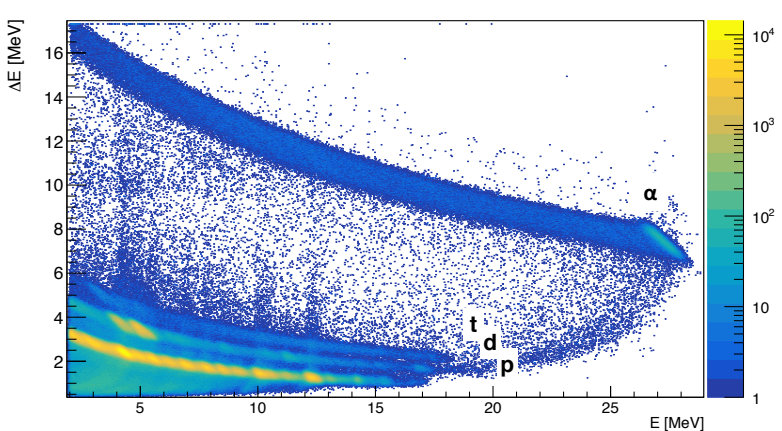

Figure 9: Particle identification plot from 35.9(2) MeV alpha particles incident on ${ }^{240} \mathrm{Pu}$. Reaction products are detected at $100^{\circ}$.

on counting statistics.

The fission fragment angular distribution associ320 ated with the alpha-fission coincidence spectrum in Figure 10 is given in Figure 11 from 6.0 to $6.5 \mathrm{MeV}$ excitation. The fission angle is measured relative to the nuclear symmetry axis; approximated as the nuclear recoil angle. The recoil angle is different for alpha particles measured at $90^{\circ}$ and $-90^{\circ}$ therefore some fission angles are covered by multiple detectors. This correction results in a large range in the associated uncertainties between angles. The fission fragment angular distribution is a function excitations of vibrational and rotational states at the saddle point of the transition nucleus. The population of these states strongly influences fission probabilities around the neutron separation energy and constrain cross section calculations [19]. Fission fragment angular distributions from the DEATH-STAR array are suited to study these states as a function of $\alpha$ scattering angle and excitation energy. With adequate statistics these states can also be studied by examining changes in the $\alpha$ spectrum as a function of fission angle.

\subsection{Signal Processing}

Each of the $E$ and $\Delta E$ detectors are individually biased and controlled from two Wiener MPOD high voltage modules. The $\Delta E$ detectors nominally operate at $200 \mathrm{~V}$, while the $E$ detectors operate at $1000 \mathrm{~V}$. The detectors are DC coupled through Swan Research Charge8V [1] preamplifiers housed in boxes located just outside the vacuum chamber. From the preamp boxes the $E$, $\Delta E$, and fission signals are connected to separate Mesytec MSCF16-LN shaping amplifiers via 34-pin ribbon-to-lemo breakout cables. Channels are individually adjusted for gain, trigger threshold, and 


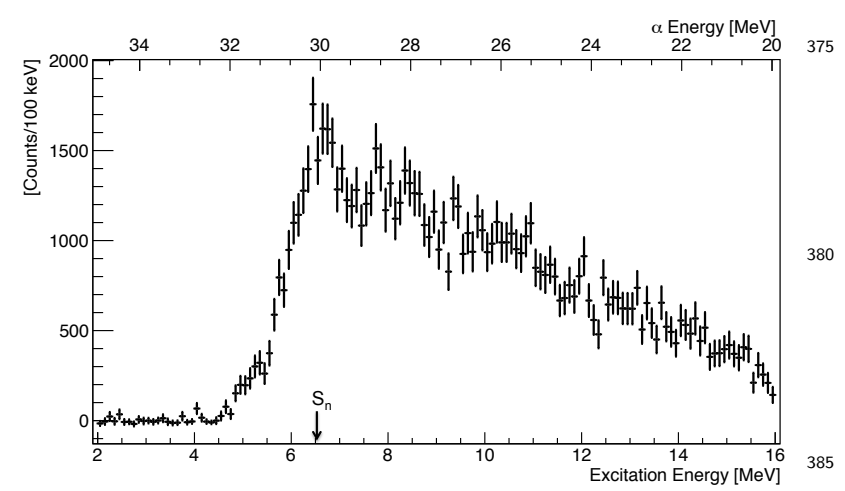

Figure 10: Alpha spectrum from the ${ }^{240} \mathrm{Pu}\left(\alpha, \alpha^{\prime} f\right)$ reaction. Scattered alphas are detected at $90^{\circ}$ and $-90^{\circ}$ in coincidence with fission.

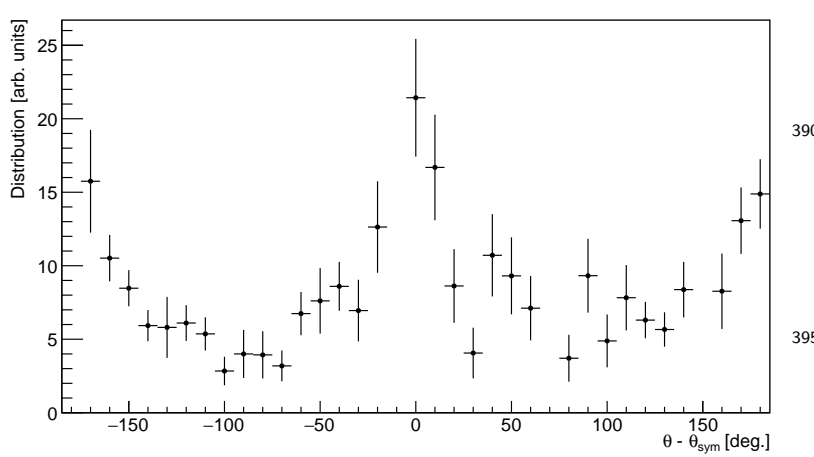

Figure 11: Angular distribution of fission fragments from the ${ }^{240} \mathrm{Pu}\left(\alpha, \alpha^{\prime} f\right)$ reaction integrated over 6.0 to $6.5 \mathrm{MeV} 400$ excitation energy.

pole-zero cancellation. Shaping times are selected in groups of four. Each shaper has three branches of output; the shaped pulse output is connected to a Mesytec MADC-32 peak sensing analog-todigital converter. The fast-timing ECL output is 405 connected to a CAEN V1190A time-to-digital converter. A NIM OR trigger across the shaper's 16 channels is used to set the system master trigger.

The $E$ shaper OR trigger is stretched to $200 \mathrm{~ns}$ width, the $\Delta E$ trigger is stretched to $50 \mathrm{~ns}$ width and delayed to arrive in the center of a coincident $E$ trigger. The master "particle" trigger is generated with the AND condition of these two pulses. It is also possible to set the master trigger from the OR trigger of the fission shapers. An artificial $25 \mu \mathrm{s}$ dead time "veto" is imposed following a master trigger to prevent new triggers during the ADC digiti- ${ }_{415}$ zation time. The real trigger rate is recorded before the dead time addition and live rate is recorded after via a STRUCK SIS3820 scaler. From the master trigger, a 4 s acceptance window is generated for 420 the ADCs; any pulse above the set ADC threshold inside of the acceptance window across all channels is recorded. The TDC trigger is delayed $400 \mathrm{~ns}$ from the master trigger. The TDC stores hit information from the ADC fast-out in on-board memory and looks "back in time" and records the time difference between shaper hits and the TDC trigger for all channels with 25 ns precision. Data buffers from the ADCs, TDC, and scaler are packaged, managed, and recorded to disk by the MIDAS 20 data acquisition system developed at TRIUMF.

\section{Conclusions}

The DEATH-STAR array provides a versatile configuration of silicon telescopes to perform particle identification at forward and backward scattering angles combined with a large segmented array of photovoltaic fission fragment detectors. The photovoltaic detectors enable efficient detection of fission fragments without sensitivity to light-ion backgrounds, essential for near-beam measurements of fission fragments and for fission measurements of isotopes with large alpha decay rates. The combination of particle and fission angular distributions will be used in the future to study angular momentum state population in compound nuclei and their associated fission probabilities.

\section{Acknowledgments}

This work was performed under the auspices of the U.S. Department of Energy by Lawrence Livermore National Laboratory under Contract DEAC52-07NA27344. This material is based upon work supported by the U.S. Department of Homeland Security under Grant Award Number 2012DN-130-NF0001. The views and conclusions contained in this document are those of the authors and should not be interpreted as representing the official policies, either expressed or implied, of the U.S. Department of Homeland Security.

\section{References}

[1] G. Siegert, Photo-voltaic Cells as Fission-Product Detectors, Nucl. Instum. and Methods 164 (3) (1979) 437438. doi:10.1016/0029-554X(79)90076-4.

[2] N. Ajitanand, R. Anand, S. Murthy, K. Iyengar, The Photovoltaic Cell - An Eminent Fission Fragment Detector, Nucl. Instrum. and Methods in Phys. A 300 (2) (1991) 354-356. doi:10.1016/0168-9002(91)90448-Y 
[3] E. Liatard, S. Akrouf, J. Bruandet, A. Fontenille, F. Glasser, P. Stassi, T. Chan, Detection of FissionProducts Using Photovoltaic Cells, Nuclear Instrum. and Methods in Phys. A 267 (1) (1988) 231-234. doi: 10.1016/0168-9002(88)90652-3

[4] C. Theisen, C. Gautherin, M. Houry, et al., SAPhIR: a fission-fragment detector, Nuclear Fission and FissionProduct Spectroscopy: Second Inernational Workshop, The American Institute of Physics, 1998, pp. 143-150.

[5] T. Ethvignot, T. Granier, L. Giot, P. Casoli, R. Nelson, A fission-fragment-sensitive target for X-ray spectroscopy in neutron-induced fission, Nucl. Instum. and

Methods A 490 (3) (2002) 559-565. doi:10.1016/ S0168-9002(02)01096-3.

[6] M. Petit, T. Ethvignot, T. Granier, R. Haight, J. O'Donnell, D. Rochman, S. Wender, E. Bond, T. Bredewe, D. Vieira, J. Wilhelmy, Y. Danon, A compensated fission detector based on photovoltaic cells, Nucl. Instum. and Methods A 554 (1-3) (2005) 340-346. doi: 440 10.1016/j.nima.2005.08.093

[7] J. J. Ressler, J. T. Burke, J. E. Escher, C. T. Angell, M. S. Basunia, C. W. Beausang, L. A. Bernstein, D. L. Bleuel, R. J. Casperson, B. L. Goldblum, J. Gostic, R. Hatarik, R. Henderson, R. O. Hughes, J. Munson, L. W. Phair, T. J. Ross, N. D. Scielzo, E. Swanberg, I. J. Thompson, M. Wiedeking, Surrogate measurement of the Pu-238(n, f) cross section, PHYSICAL REVIEW C 83 (5). doi: $\{10.1103 /$ PhysRevC.83.054610\}

[8] C. Hsieh, P. Murley, R. O'Brien, Collection of Charge from Alpha-Particle Tracks in Silicon Devices, IEEE Transactions on Electron Devices 30 (6) (1983) 686693.

[9] C. Hsieh, P. Murley, R. O'Brien, A Field-Funneling Effect on the Collection of Alpha-Particle-Generated Carriers in Silicon Devices, Electron Device Letters 2 (4) (1981) 103-105.

[10] F. McLean, T. Oldham, Charge Funneling in N-Type and P-Type Si Substrates, IEEE Transactions on Nucl. Science 29 (6) (1982) 2018-2023.

[11] Swan research (May 2016). URL swanresearch. xorgate.com

[12] H. Schmitt, F. Pleasonton, Evaluation of Semiconductor Detectors for Fission Fragment Energy Measurements, Nucl. Instum. and Methods 40 (2) (1966) 204-\&. doi:10.1016/0029-554X(66)90373-9

[13] C. Boiano, R. Bassini, A. Pullia, A. Pagano, Widedynamic-range fast preamplifier for pulse shape analysis of signals from high-capacitance detectors, IEEE Transactions on Nuclear Science $51(5,1)$ (2004) 1931-1935. doi:10.1109/TNS.2004.832308

[14] Ortec, L Series Room Temperature Lithium-Drifted Silicon Detector, datasheet 051512 (May 2016).

[15] Ortec, B Series Totally Depleted Silicon Surface Barrier Detectors, datasheet 052412 (May 2016).

[16] S. Lesher, L. Phair, et al., STARS/LiBerACE: Segmented silicon and high-purity germanium detector arrays for low-energy nuclear reaction and structure studies, Nuclear Instrum. and Methods in Phys. A 621 (2010) 286-291.

[17] C. Dunford, T. Burrows, Online nuclear data service, Report IAEA-NDS-150 (NNDC Informal Report NNDC/ONL-95/10) Rev. 95/10.

[18] F. Goulding, D. Lands, J. Cerny, R. Pehl, A new particle identifier technique for zeta $=1$ and zeta $=2$ parti-
II Instr. and Meth. A 31 (1) (1964) 1-12. doi:\{10.1016/ 0029-554X (64)90313-1\}.

[19] W. Younes, H. C. Britt, Neutron-Induced Fission Cross Sections Simulated rom $(t, p f)$ Results, Physical Review C 67 (2), feb. doi:10.1103/PhysRevC.67.024610

[20] TRIUMF, MIDAS: Maximum Integrated Data Acquisition System, https://bitbucket.org/tmidas/midas. 